\title{
Barreras en la comunicación asistencial con pacientes inmigrantes de origen amazig
}

\section{Healthcare communication barriers with amazigh immigrant}

\author{
Carla Ferrerós Pagèsa, Mònica Barrieras Angàsb, Francesc Roca Urgella, Alícia Baltasar Baguéc \\ a Grup de Lèxic i Gramàtica (GLG), Departament de Filologia i Comunicació, Facultat de Lletres, Universitat de Girona, España \\ b Grup d'Estudi de Llengües Amenaçades (GELA), Departament d'Educació Lingüística i Literària, i Didàctica de les Ciències \\ Experimentals i de la Matemàtica, Universitat de Barcelona, España \\ c Grup de Lèxic i Gramàtica (GLG), Departament d'Infermeria, Facultat d'Infermeria, Universitat de Girona, España
}

\section{Resumen}

Introducción: Estudio sobre problemas de comunicación asistencial teniendo en cuenta la institución y la lengua y cultura de los pacientes. Se estudiará la expresión de síntomas relacionados con cuestiones no orgánicas desde el punto de vista lingüístico, y que genera malentendidos que suelen pasar desapercibidos por los profesionales. Objetivos: El objetivo principal es analizar barreras de comunicación con pacientes de origen amazig. Los objetivos específicos son analizar recursos disponibles y formación relacionada con la atención a pacientes inmigrantes y describir dificultades de comunicación. Metodología: Cualitativa descriptiva. Realizado en los centros de atención primaria y hospitales de las comarcas de Girona y Barcelona escogidos al azar. Participantes: 32 informantes (13 médicos, 9 enfermeros, 4 mediadores y 6 trabajadores sociales). Se utilizó un cuestionario y una entrevista semiestructurada. El análisis de los discursos e interpretación de los contenidos se realizó con el soporte Atlas.ti 6.0. Resultados: Los profesionales refieren una distribución desigual de los pacientes inmigrantes y carencia de recursos y de tiempo. Las dificultades de comunicación son no son detectadas en la mayoría de los casos. Conclusiones: Algunas cuestiones lingüísticas pueden dificultar la comunicación. Más recursos y formación proporcionada por la institución podrían ayudar a solventar este problema.

Palabras clave: barreras de comunicación; diversidad cultural; inmigración; servicios de salud; atención primaria; idioms of distress.

\section{Abstract}

Introduction: Study about communication problems taking into account issues related to the institution and to patients' language and culture. The expression of symptoms related to non-organic issues will be studied from a linguistic point of view as it generates misunderstandings that may go unnoticed by professionals. Objectives: The main objective is to analyse the communication barriers with immigrant patients of Amazigh origin. Specific objectives: analyse the available resources and the training in relation to the care immigrant patients receive; detailing strictly linguistic difficulties. Methodology: Qualitative. Descriptive. Setting: Hospitals and primary health care centers in Girona and Barcelona areas have been chosen at random. Participants: 32 informants: 13 doctors, 9 nurses, 4 intercultural mediators and 6 social workers. Process followed: A survey and a partially structured interview. For the analysis of the conversations and the interpretation of their content Atlas.ti 6.0 software was employed. Results: The healthcare professionals detected an unequal distribution and a lack of resources and time. Possible communication difficulties go undetected in the majority of cases. Conclusions: Some linguistic aspects may make the communication between healthcare professionals and patients speaking different L1 difficult. More resources and training coming from healthcare institutions would help solve this problem.

Key words: barrier communication; cultural diversity; immigration; health services; primary care; idioms of distress. 


\section{Introducción}

$\mathrm{D}$ esde finales del siglo pasado coexisten en España un gran número de lenguas. En Cataluña, el colectivo inmigrante más numeroso es el formado por personas de procedencia marroquí con un total de 211.192 individuos, representando el $19,52 \%$ respecto el total de la población extranjera (Institut d'Estadistica de Catalunya, 2018), la mayoría de las cuales tienen el amazig como primera lengua (L1) (Barrieras, 2014). La coexistencia de diferentes lenguas suele ocasionar dificultades de intercomprensión entre profesionales de la salud y pacientes (Crous, Gràcia y Baltasar, 2015), creándose una barrera de comunicación intercultural que tiene consecuencias negativas en el diagnóstico, tratamiento y pronóstico del paciente (Gailly, 1997) y que puede ocasionar problemas médicamente significativos (Murray y Skull, 2005). Además, afecta la toma de decisiones, la planificación y la capacidad de proporcionar servicios de salud eficaces a las comunidades con lenguas, circunstancias migratorias y estatus socioeconómicos diferentes (Chu,1998; Bowen, 2000).

Estas cuestiones han sido estudiadas desde diferentes disciplinas. En el ámbito de las ciencias de la salud, no obstante, existen pocas investigaciones sobre comunicación asistencial como se señala en la revisión bibliográfica realizada por López (2010), en especial el artículo dedicado a la asistencia de grupos marginalizados entre los cuales se puede contar la población inmigrante. Pero se han estudiado sobre todo desde disciplinas como la antropología y la comunicación intercultural (Comelles y Martínez, 1993; Almagro et al., 2010; Llosada, Vallverdú, Miró, Pijem y Guarga, 2012).

La expresión de los síntomas que hacen referencia a cuestiones no orgánicas puede tener significados distintos para los pacientes y profesionales que no hablan una misma lengua. Por ejemplo, los llamados idioms of distress se refieren a la expresión cultural de las enfermedades y tienen significados interpersonales y sociales amplios: explicitan síntomas somáticos y, si el profesional los interpreta incorrectamente, pueden llevar a procedimientos y tratamientos innecesarios 0 inadecuados (Desai y Chaturvedi, 2017). En un estudio realizado por Tamarit, Gallegos, Amela y Radu (2009) se señala que, cuando la comunicación entre profesional y paciente se ve afectada por el idioma, los profesionales se sienten insatisfechos y frustrados por la comunicación con los pacientes inmigrantes en contextos determinados.

Hay pocos estudios que analicen la comunicación asistencial desde la perspectiva del léxico y la semántica (Crous, et al., 2016; Gràcia, Ferrerós y Baltasar, 2017; Ferrerós, 2019). Cada lengua muestra una manera distinta de segmentar y ordenar realidad. La categorización es un proceso cognitivo que agrupa y ordena las entidades y eventos de la realidad en función de rasgos relevantes (Taylor, 2002) y se refleja lingüísticamente en maneras diferentes de ordenar la realidad. Las lenguas muestran diferencias léxicas que pueden ocasionar malentendidos incluso cuando los interlocutores hablan la misma lengua. La polisemia es un fenómeno que muestra diferencias interlingüísticas importantes: es frecuente que las palabras para partes del cuerpo tengan más de un significado que pueden no coincidir interlingüísticamente (Ibarretxe-Antuñano,1999; Ibarretxe-Antuñano, 2010). Las expresiones polisémicas son frecuentes en los idioms of distress, que ponen de manifiesto diferencias de codificación de significados mediante una misma palabra (Nitchter, 2010; Ferrerós, 2019).

Partimos de la hipótesis de que existen dificultades de comunicación, no detectadas por parte de los profesionales, derivadas de las diferenciasinterlingüísticas de categorización y de asignación de significados no literales a palabras que designan partes del cuerpo y que tales dificultades son agravadas por la falta de recursos y de formación en los centros asistenciales. A pesar de que el estudio es eminentemente lingüístico, consideramos que hay otras cuestiones que pueden interferir en el proceso comunicativo. Es por este motivo que, para conseguir el objetivo principal del estudio, analizar las barreras de comunicación con pacientes amazigs, nos planteamos dos objetivos específicos: 1) describir y analizar los recursos disponibles y la formación por parte de los profesionales; es decir, analizar aquellos aspectos relacionados con la comunicación con pacientes inmigrantes que atañen a la institución (subobjetivo 1) y 2) describir y analizar las dificultades de comunicación con un colectivo de pacientes inmigrantes concreto y muy presente en Cataluña, el que tiene como L1 la lengua amazig, derivadas de las diferencias de categorización y denominación de las partes del cuerpo y enfermedades (subobjetivo 2). Nos centramos en un solo colectivo por la imposibilidad de estudiar todas las dificultades lingüísticas de todas las comunidades atendidas.

\section{Participantes y métodos}

\section{Diseño y contexto}

Estudio cualitativo descriptivo realizado en Centros de Atención Primaria (CAPs) y hospitales de las comarcas 
de Girona y Barcelona escogidos al azar: Martorell, Banyoles, Sarrià de Ter, Celrà, Salt, Anglès, Arbúcies, Girona y Sant Sadurní (años 2015-18).

\section{Muestra y participantes}

El muestreo teórico fue la estrategia para escoger a los participantes. La selección de los participantes fue intencional, no aleatoria y no finalizó hasta alcanzar la saturación. Los criterios de inclusión fueron:

1.Profesión: médicos, enfermeros, mediadores o trabajadores sociales.

2. Sexo: esta variable puede influir en la obtención de información relacionada con temas que pueden ser considerados tabúes en ciertas culturas.

3.Lengua: se seleccionaron profesionales con L1 español y catalán y profesionales con L1 amazig (es decir, compartida con los pacientes que tenemos en cuenta en este trabajo).

La muestra está compuesta por 32 informantes: 13 médicos ( 9 mujeres, 4 hombres), 9 enfermeros (7 mujeres, 2 hombres), 4 mediadoras y 6 trabajadores sociales (4 mujeres, 2 hombres); 24 informantes, pues, son mujeres y 8 son hombres. Hay 2 mediadores, 2 trabajadores sociales, 1 médico y 1 enfermero que tienen el amazig o el árabe como L1. La presencia de las dos lenguas se justifica porque, al tratarse de lenguas en contacto con grandes interferencias mutuas y habladas por comunidades que comparten rasgos culturales, los profesionales con $\mathrm{L} 1$ árabe proporcionan información relevante. A cada participante se le solicitó el consentimiento informado para la grabación de los discursos. A cada informante le corresponde una etiqueta compuesta por el nombre de su profesión y un número que hace referencia al orden de realización de las entrevistas. Así, por ejemplo, la etiqueta "Enfermera 3" designa a la tercera enfermera entrevistada.

\section{Obtención de los datos}

1. En relación con los datos sobre la comunicación con pacientes inmigrantes que atañen a la institución (subobjetivo 1), se utilizó un cuestionario sobre aspectos relacionados con la institución: número, procedencia y L1 de los pacientes, los recursos para facilitar la comunicación, el tipo de formación recibida relacionada con la atención a pacientes inmigrantes y la existencia de un protocolo de atención.

2. En relación con los datos estrictamente lingüísticos y culturales (subobjetivo 2), se realizó una entrevista semiestructurada sobre las posibles dificultades lingüísticas y culturales que llevan a una mala comprensión entre el paciente inmigrante y el profesional de la salud, estructurada en dos partes: 1) diferencias de categorización a partir de ejemplos expuestos por el entrevistador y 2) diferencias de asignación de significados a palabras polisémicas mediante procesos de metáfora o de metonimia.

\section{Análisis de los datos}

1. Lectura en profundidad para extraer las unidades de significado más relevantes que emergían de los discursos. 2. Agrupación en categorías que aglutinaban aquellas unidades de significado más pequeñas que tenían algo en común.

3. Interpretación de los contenidos de cada categoría mediante Atlas ti 6.0 .

\section{Resultados}

En este apartado se analizarán resultados relacionados con dos categorías principales correspondientes a los dos subobjetivos del estudio.

\section{Institución}

\section{Organización institucional}

Los profesionales entrevistados indicaron el número de pacientes inmigrantes atendidos diariamente (en la tabla 2 no incluimos los mediadores interculturales, ya que, debido a la naturaleza de su trabajo todos los pacientes que atienden son de origen extranjero) (Ver tabla 2).

En lo que hace referencia a la formación, 11 de los profesionales entrevistados afirman que no han recibido ningún tipo de formación relacionada con la atención a pacientes inmigrantes: 5 médicos/-as, 2 enfermeros/-as y 4 trabajadores/-as sociales. Dejamos de lado las mediadoras interculturales, que tienen una formación específica para la atención a las personas de cada comunidad.

La mayoría de la formación recibida por el resto de profesionales gira en torno a las siguientes cuestiones no lingüísticas: diabetes y Ramadán; mutilación genital femenina; y/o enfermedades tropicales y vacunación.

Solo una enfermera (Banyoles) y un médico (Montilivi) mencionan un curso de comunicación verbal y no verbal, y 3 profesionales de Martorell aluden a sesiones de atención al inmigrante centradas en el protocolo de actuación. 
Dos de los trabajadores/-as sociales entrevistados cultural). Algunos de los profesionales reclaman también también tienen formación específica en interculturalidad, la necesidad de formación específica para profesionales pero no ofrecida por la institución (por ejemplo, uno de o conferencias impartidas por personas de cada país de ellos se refiere su licenciatura en antropología social y origen de las comunidades mayoritarias.

Tabla 1. Categorías, subcategorías, barreras y facilitadores.

\begin{tabular}{|c|c|c|c|}
\hline Categorías & Subcategorías & Facilitador & Barrera \\
\hline Institución & Organización & $\begin{array}{l}\text { Distribución desigual de } \\
\text { inmigrantes } \\
\text { Bajo número de pacientes } \\
\text { Formación lingüística y } \\
\text { cultural } \\
\text { Protocolo consensuado }\end{array}$ & $\begin{array}{l}\text { Distribución desigual de } \\
\text { inmigrantes } \\
\text { Alto número de pacientes } \\
\text { Falta de formación } \\
\text { lingüística y cultural } \\
\text { Falta de protocolo } \\
\text { consensuado }\end{array}$ \\
\hline & Gestión económica & $\begin{array}{l}\text { Más tiempo asistencial } \\
\text { Equipos cualificados } \\
\text { Presencia del mediador } \\
\text { Disponibilidad de recursos } \\
\text { útiles }\end{array}$ & $\begin{array}{l}\text { Falta de tiempo asistencial } \\
\text { Falta de espacios } \\
\text { Ausencia de recursos útiles }\end{array}$ \\
\hline $\begin{array}{l}\text { Comunicación } \\
\text { profesionales- } \\
\text { pacientes }\end{array}$ & $\begin{array}{l}\text { Categorización y } \\
\text { denominación } \\
\text { Diferencias en la } \\
\text { asignación de significados } \\
\text { no literales }\end{array}$ & $\begin{array}{l}\text { Misma L1 profesional - } \\
\text { paciente } \\
\text { Uso de otras lenguas } \\
\text { compartidas } \\
\text { Escolarización del paciente } \\
\text { en país de acogida }\end{array}$ & $\begin{array}{l}\text { Diferente L1 profesional - } \\
\text { paciente } \\
\text { Desconocimiento de } \\
\text { fenómenos lingüísticos/ } \\
\text { culturales }\end{array}$ \\
\hline
\end{tabular}


Tabla 2. Número y porcentaje de pacientes de origen extranjero atendidos al día.

\begin{tabular}{|lccc|}
\hline & Médicos/-as & Enfermeros/-as & Trabajadores/-as Sociales \\
\hline Más de 30 & 1 & 1 & - \\
Entre 25 y 29 & 5 & 3 & - \\
Entre 20 y 24 & - & - & - \\
Total, $\mathrm{N}=$ & $\mathrm{N}=6(46,15 \%)$ & $\mathrm{N}=4(44,4 \%)$ & - \\
Entre 15 y 19 & - & - & 1 \\
Entre 10 y 14 & 2 & 1 & $\mathrm{~N}=1(16,67 \%)$ \\
Total, $\mathrm{N}=$ & $\mathrm{N}=2(15,38 \%)$ & $\mathrm{N}=1(11,11 \%)$ & 3 \\
Entre 5 y 9 & 5 & 4 & 2 \\
Menos de 5 & - & - & $\mathrm{N}=2(83,33)$ \\
Total, $\mathrm{N}=$ & $\mathrm{N}=5(38,46 \%)$ & $\mathrm{N}=4(44,44 \%)$ & 6 \\
Total, $\mathrm{N}=28$ & 13 & 9 & 6 \\
\hline
\end{tabular}

\section{Gestión económica}

25 de los participantes creen que el equipo, los materiales y el tiempo del que disponen no son suficientes o son poco ágiles. Solo un médico cree que es mejor no utilizar los servicios de un mediador: «El mediador impide el contacto directo, y hay que tener en cuenta que el contacto directo ayuda a no alterar la información que nos da el paciente» (Médico 3). El resto de participantes creen que más mediadores interculturales y más tiempo de atención mejoraría el servicio ofrecido. Para suplir la falta de recursos, se utiliza material de apoyo: traductor de Google, Doctor Speaker, dibujos y mímica.

Todos los participantes afirman que hay pacientes que van acompañados por un amigo o un familiar en la consulta que actúa de traductor, y que, a pesar de ciertos inconvenientes (privacidad, confidencialidad, en muchos casos son niños, etc.) esto agiliza la consulta y facilita la comunicación.

Los 7 profesionales que afirman que los recursos son suficientes se dividen en dos categorías. 4 forman parte del grupo que atiende menos pacientes inmigrantes y afirman que pueden dedicarles tiempo. Los otros no tienen la sensación de que haya problemas de intercomprensión, a pesar de que las mediadoras interculturales señalan que existen dificultades no percibidas por parte del médico 0 enfermero, como veremos en el punto siguiente.

\section{Comunicación entre profesionales y pacientes}

\section{Diferencias de categorización}

Solo una enfermera (de los profesionales con L1 catalán o castellano) afirma que ha tenido problemas de comunicación derivados de diferencias de categorización: «Tienen expresiones diferentes (...) y me ha pasado que no las he entendido y entonces les digo, 'que te refieres a...'. Entonces ya me lo explican de otra manera, porque claro, las expresiones son diferentes.» (Enfermera 7).

Los profesionales entrevistados de L1 amazig o árabe sí son conscientes de las dificultades derivadas de las diferencias de categorización en las lenguas implicadas. Las mediadoras interculturales, al tener una alta competencia en ambas lenguas, detectan dificultades que pasarían desapercibidas:

A pesar de que haya muchos pacientes que hablen catalán o español hay cosas que a veces no entienden. Dicen que sí, pero salen de allí sin idea de lo que les ha dicho el médico. A veces no encontramos la palabra exacta para explicarle al paciente 0 al profesional. A veces, el profesional te dice algo que entiendes, pero cuesta decirle al paciente qué quiere decir. No hay algunas palabras exactamente iguales, que den el mismo significado que dicen los dos (Mediadora 2). 
En estas ocasiones pueden recurrir al francés cuando el paciente ha estado escolarizado en su país de origen, especialmente para referirse a enfermedades. Si los pacientes no están familiarizados con el francés 0 el inglés, los mediadores utilizan expresiones no técnicas y, por lo tanto, no coincidentes con las de la biomedicina occidental:

Me encuentro con muchos problemas de traducir enfermedades. Por ejemplo, si me dicen una neumonía y si la persona no entiende qué es, pues le digo: "¿sabes? El pulmón está enfermo" y ya está. La tuberculosis sería la "enfermedad del pulmón" (Mediadora 1).

Un médico con el árabe como L1 señala que en su lengua las enfermedades pueden etiquetarse con expresiones que explicitan alguno de los síntomas que producen, y una traducción literal puede conllevar malentendidos. Algunos de los ejemplos que menciona son del árabe, pero tienen expresiones equivalentes en amazig:

Una persona tiene una hernia y lo dice así: "que tengo un poco el vientre abierto". O para decir que tiene un dolor de espalda, si hay una infección, dice: "el síntoma que tengo es que tengo aire en la espalda, tengo aire". "Tengo un poco abierta la espalda (...) y necesito que alguien la cure y la cierre". O, por ejemplo (...) la varicela, la gente mayor dicen que es la "roja" (...) porque antes las abuelas ponían un pañuelo a los niños, un pañuelo rojo para que no los cogiese un brujo. (...) Porque saben que es una enfermedad que el niño puede morir, porque antes sí se morian (...). Y ha quedado este tópico y lo cuentan así: "iya tiene la roja!" (...) También nosotros decimos "el perro", que es la laringitis. Tiene la enfermedad del perro (Médico 12).

\section{Diferencias en la asignación de significados no literales}

Algunos de los participantes afirman que muchos pacientes procedentes de Marruecos se quejan de dolor de cabeza y lo atribuyen a distintas causas:

Nos encontramos que hay muchos problemas de... de dolor. El umbral del dolor que tienen... parece que está más abajo» [o bien] «Supones que es un tema de estrés, pero cuando lo abordas, no lo asumen (...). Y al final ves que no hay una patología orgánica y piensas más en causas psicológicas. Pero no lo aceptan (...). Para ellos es un dolor de cabeza (Enfermera 1).

No solo el dolor de cabeza, sino sensaciones asociadas al corazón son referidas por los pacientes de origen marroquí, según los profesionales entrevistados:

Una niña pequeña vino un día con su madre, "me duele el corazón, me duele el corazón", y la madre (...) le buscó una visita al CAP y dice que no tiene nada en el corazón, tenía problemas de estómago (Médico 9).

En lo que hace referencia a las enfermedades no orgánicas, el médico con L1 árabe ha detectado que algunos pacientes hacen una descripción de los síntomas derivada de la asociación de significados no literales a partes del cuerpo distinta a la que se hace en catalán 0 en español:

"Me duele el corazón", por la tristeza. O "siento que el hígado me estalla". De la tristeza, de estar enfadado. Puede ser que vengan diciendo que les duele el hígado y es un dolor emocional, pero lo somatizan así. "Tengo la cabeza que me da vueltas", también de una emoción. 0 "veo doble, no veo bien, veo a aquella persona doble", de tanto dolor emocional que le ha producido (Médico 12).

También afirma que los amazigófonos de más edad explican los síntomas psíquicos como físicos:

Ellos vienen asi y no te dicen... "tengo problemas, estoy triste". Vienen y dicen: "mira, tengo algo que me aprieta el pecho y me ahoga". Tú vas preguntando y llegas al punto. (...) Algunos dicen: "mira, tengo algo como si estoy poseído, tengo algo en la cabeza y después me deja ciego". Es una migraña con aura (Médico 12).

\section{Discusión}

En primer lugar, tendremos en cuenta los resultados relativos a la institución. Más allá de analizar la percepción que tienen los profesionales en lo que hace referencia a las dificultades lingüísticas de comunicación con un colectivo de pacientes de origen inmigrante, consideramos imprescindible describir también aquellos aspectos que dependen de la institución y que puedan tener una incidencia en la comunicación asistencial, como son los recursos disponibles y la formación recibida por parte de los profesionales. Otros estudios también relacionan el papel de la institución con la atención a los pacientes inmigrantes y las dificultades que puede conllevar el hecho de no compartir la lengua o aspectos culturales con los profesionales de la salud que los atienden (Tamarit et al., 2009).

En lo que hace referencia a la organización institucional y en relación con el número de pacientes atendidos por cada profesional, se observa que algunos atienden un alto número de pacientes inmigrantes y otros que atienden un número bajo, pero no hay casos intermedios. Esto se debe a la distribución desigual de la población en las zonas urbanas analizadas. Los profesionales que atienden menos pacientes inmigrantes son los de los 
CAP de Montilivi (Girona), Martorell y Sant Sadurní.

Tampoco hemos visto uniformidad respeto a la formación recibida por los profesionales: gran parte de ella gira entorno a cuestiones no lingüísticas. Los profesionales más formados en comunicación y atención a los inmigrantes son aquellos que trabajan en CAP con un número menor de pacientes extranjeros.

Los profesionales hacen referencia a la falta de recursos (mediadores interculturales y mayor tiempo de atención). A veces la presencia de un familiar 0 amigo del paciente facilita la comunicación, aunque hay inconvenientes asociados como la falta de privacidad y la confidencialidad (Seguí, 2005). Los profesionales afirman que, para suplir la falta de recursos, usan traductores automáticos, dibujos y mímica. Pero esto conlleva varios problemas: el traductor de Google y programas similares requieren que el paciente sepa leer en su L1; la variedad recogida por los traductores es la estándar, desconocida por hablantes de variedades del árabe 0 del chino, por ejemplo; y algunas L1 de los pacientes no están contempladas. En el caso de la mímica, hay que señalar que muchos gestos no son universales y pueden provocar más problemas de comunicación (Vázquez, Terraza, Vargas y Lizana, 2009; Crous et al., 2016).

En lo que hace referencia a la comunicación entre profesionales y pacientes de L1 amazig, los mediadores interculturales afirman que algunas veces les es difícil traducir expresiones utilizadas por los profesionales de la salud. En algunos casos, recurren al francés. El hecho de que en Marruecos la lengua vehicular de los estudios científicos sea el francés o el inglés (Ennaji, 2005; Chaker, 2013) ha causado que las lenguas propias del territorio, el árabe marroquí y el amazig, no hayan accedido a este ámbito de uso. Cuando los pacientes no conocen el francés, los mediadores interculturales y los profesionales que comparten L1 con ellos utilizan expresiones no coincidentes con las de la biomedicina occidental. En este sentido, si en la consulta no hay un mediador intercultural y el profesional no tiene la misma L1 que el paciente, es posible que no se perciba que no hay una buena comprensión del discurso.

Otros problemas de comunicación se relacionan con la diferente asignación de significados a palabras para partes del cuerpo en las lenguas implicadas, los idioms of distress (Nitchter, 2010). Así, por ejemplo, el hecho de que muchos pacientes de origen marroquí se refieren a menudo al dolor de cabeza o a síntomas asociados con el corazón se relaciona con los diferentes significados que tienen estas palabras en amazig. Las partes del cuerpo que se suelen asociar con el dolor son designadas con palabras polisémicas: 'cabeza', 'corazón', 'hígado', etc. Los significados no centrales de estas palabras en amazig no coinciden con los significados de las palabras equivalentes en catalán o español, pero los pacientes se los atribuyen al hablar la L2. En amazig, azllif, que tiene como significado principal 'cabeza', también codifica significados relacionados con el estrés o los nervios: "Cuando tienes problemas y estás muy estresado, tienes muchos nervios, dices en amazig "me explota la cabeza". El estrés, los nervios, cuando no das abasto o cuando no estás bien, eso se pone en la cabeza" (Ferreros, 2019). Una traducción literal de esta expresión genera malentendidos en la consulta. Igualmente, la palabra ul, que en amazig significa literalmente 'corazón', por un proceso metonímico también puede designar 'estómago' en expresiones como iteqsa-yi ul inu (literalmente, 'me duele el corazón', 'tengo náuseas') (Taifi, 1996; Serhoual, 2002; El Adak, 2006). Este ejemplo explica las palabras reproducidas en el apartado anterior del profesional que refería el ejemplo de la adolescente con problemas gástricos.

Este estudio no está exento de limitaciones. El ámbito de estudio es relativamente reducido y no hay una distribución equilibrada de las variables tenidas en cuenta para la selección de participantes, en esta línea, hemos contado con más mujeres que hombres, y con más profesionales con L1 catalán o español que profesionales con L1 amazig.

\section{Conclusiones}

Respecto a la institución, se observa una distribución desigual de la población extranjera en los centros donde trabajan los profesionales entrevistados. Es relevante que los más formados en cuestiones de comunicación y atención a los pacientes inmigrantes son los que trabajan en centros con menos pacientes extranjeros.

Los recursos (equipo, materiales y/o tiempo) son escasos y poco agiles. Para suplir estas carencias, los profesionales utilizan material de apoyo que no resulta suficiente: el desconocimiento de las diferencias interlingüísticas de las lenguas habladas por los pacientes o de la no universalidad de la gestualidad pueden ocasionar malentendidos no detectados por profesionales y pacientes. Algunos profesionales se refieren al hecho de que hay pacientes que acuden a la consulta acompañados por amigos o familiares con más dominio de las L2. Aun así, este hecho conlleva ciertos inconvenientes, el más destacable de los cuales es la falta de privacidad.

Los mediadores afirman que las diferencias de 
significado de las expresiones lingüisticas pueden ocasionar malentendidos no detectados por parte de médicos y enfermeros. Este trabajo ha puesto de manifiesto que algunas cuestiones lingüisticas, como las diferencias de categorización y de asignación de significados no literales a palabras para partes del cuerpo, pueden impedir la comunicación entre los profesionales y los pacientes incluso cuando ambos se expresan en la lengua de la comunidad de acogida.

Agradecimientos

A Berta Crous y Adrià Mayans la realización y transcripción de algunas de las entrevistas.

Financiación

Este trabajo ha sido financiado por los siguientes proyectos: Variación microparamétrica y cambio lingüístico en morfologia, sintaxis y discurso (FFI2017-87140-C4-2-P) (MINECO) y Grup de lèxic i gramàtica (2017SGR1194) (AGAU).

\section{Referencias bibliográficas}

Almagro, M. J., Rojas, M. J., Guix, J., Garreta, S., Ribot, B., \& Arija, V. (2010). Percepción del sistema sanitario catalán de la población marroquí. Creencias sobre salud y enfermedad. Atención Primaria, 42(5), 266-272. https://doi.org/10.1016/j. aprim.2009.09.019

Barnieras, M. (2013). La cruilla del multilingüisme: Les llengües dels catalans al segle XXI. Divèrsia, 3 .

Bowen, S. (2000). Language barriers in access to health care. Ottawa, Ontario: Health Canada.

Comelles, J.M. y Martinez, A.M. (1993). Enfermedad, cultura y sociedad: un ensayo sobre las relaciones entre la antropología social y la medicina. Madrid, España: Eudema.

Crous, B., Gràcia, L., y Baltasar, A. (2016). El corazón y la palabra: diferencias interlingüísticas y sus consecuencias en el ámbito de la salud. Formación Médica Continuada en Atención Primaria, 23(1), 9-14. https://doi.org/10.1016/J.fmc.2015.04.008

Chaker, S. (2013). L'officialisation de tamazight (Maroc/ Algérie): quelques réflexions et interrogations sur une dynamique aux incidences potentielles considérables. Asinag, 8, 35-50.

Chu, C. (1998). Crossicultural health issues in contemporary Australia. Ethnicity \& Health, 3(1-2), 125-134. https $/ /$ doi.org/10.1080/13557858.1998.9961854
Desai, G. y Chaturvedi, S.K. (2017). Idioms of Disstres. Journal of Neurosciencies in Rural Practice, 8 (Supl. 1), pp 94-97. DOI: 10.4103/jnrp.jnrp_235_17.

Desai, G., y Chaturvedi, S. K. (2017). Idioms of Distress. Journal of Neurosciences in Rural Practice, 8(Suppl 1), 94-97. https://doi.org/10.4103/jnrp. jnrp_235_17

ElAdak, M. (2006). Le figement lexical en rifain: étude des locutions relatives au corps humain (Tesis Doctoral). Institut National de langues et civilisations Orientales de París.

Ennaji, M. (2005). Multilingualism, cultural identity, and education in Morocco. New York : Springer Science \& Business Media.

Ferrerós, C. (2019). El cuerpo y las enfermedades en catalán por parte de hablantes de rifeño: Cuestiones léxicas y semánticas en la comunicación asistencial. En N. Rodríquez y B. Heinsch (Eds.), Contextos multilingües. Mediadores interculturales, formación del profesorado de lenguas extranjeras (pp. 221-242). Bern: Peter Lang.

Gailly, A. (1997). Turkish immigrants in Belgium. En I. AlLissa y M. Tousignan (Eds.), Ethnicity, Immigration, and Psychopathology (pp. 147-164). New York: Plenum Press.

Gràcia, L., Ferrerós, C., y Baltasar, A. (2017). El cuerpo y la palabra: diferencias interlingüísticas y sus consecuencias en el ámbito de la salud. Formación Médica Continuada en Atención Primaria, 24(6), 312-318. https://doi.org/10.1016/j.fmc.2015.03.046

Ibarretxe-Antuñano, I. (1999). Polysemy and Metaphor in Perception Verbs: A Cross-Linguistic Study. (Tesis doctoral). University of Edinburgh.

Ibarretxe-Antuñano, I. (2010). Lexicografia y Lingüistica Cognitiva. Revista Española de Lingüística Aplicada, 23, 195-213.

Institut d'Estadistica de Catalunya. (2018). Padró municipal d'habitants. Recuperado 4 de septiembre de 2019, de https:/www.idescat.cat/ pub/?id=pmh

López, M.M. (2010). Comunicación Sanitaria y marginalidad. Revista Española de Comunicación en Salud, 1(2), 178-186. 
Llosada, J., Vallverdú, I., Miró, M., Pijem, C., y Guarga, A. (2012). Acceso y uso de los servicios sanitarios por parte de los pacientes inmigrantes: la voz de los profesionales. Atención Primaria, 44(2), 82-88. https://doi.org/10.1016/j.aprim.2010.11.014

Murray, S. B., y Skull, S. A. (2005). Hurdles to health: immigrant and refugee health care in Australia. Australian Health Review, 29(1), 25-29. https:// doi.org/10.1071/AH050025

Nichter, M. (2010). Idioms of distress revisited. Culture Medicine and Psychiatry, 34(2), 401-416. https:// doi.org/10.1007/s11013-010-9179-6

Seguí, M. (2005). El inmigrante en la consulta del médico de cabecera. Medicina de Familia. Semergen, 31(11), 505-507. https://doi.org/10.1016/S11383593(05) $72980-4$

Serhoual, M. (2002). Dictionnaire tarifit-français (Tesis Doctoral). Université Abdelmalek Essaâdi, Faculté des Lettres et des Sciences Humaines, Tétouan.

Taïfi, M. (1996). Étude sémantique comparative du terme "coeur" en arabe dialectal (qelb) et en berbère (ul). Études et Documents Berbères, (14), 153-162.

Tamarit, I., Gallegos, D., Amela, R., y Radu, I. (2009). Problemas éticos en la comunicación con los pacientes inmigrantes infecciosos de larga duración y con tratamientos paliativos: Informe final. Valencia: Universidad de Valencia.

Taylor, J.R. (2002). Linguistic Categorization. New York: Oxford Textbooks in Linguistics.

Vázquez, M. L., Terraza, R., Vargas, I., y Lizana, T. (2009). Necesidades de los profesionales de la salud en la atención a la población inmigrante. Gaceta Sanitaria, 23(5), 396-402. 